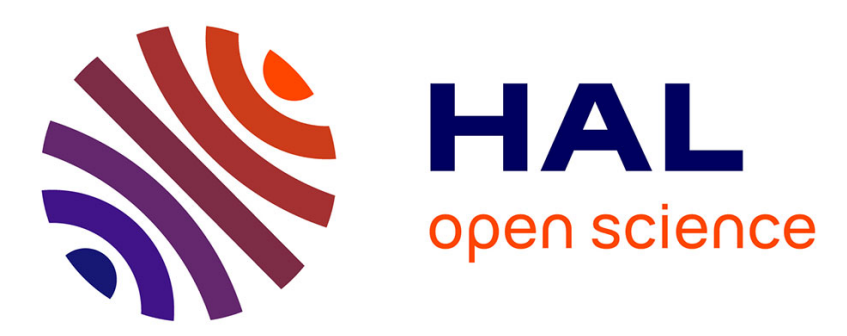

\title{
Multiparticle reaction noise characteristics
}

Zoran Konkoli

\section{- To cite this version:}

Zoran Konkoli. Multiparticle reaction noise characteristics. Journal of Theoretical Biology, 2011, 271

(1), pp.78. 10.1016/j.jtbi.2010.11.029 . hal-00657587

\section{HAL Id: hal-00657587 \\ https://hal.science/hal-00657587}

Submitted on 7 Jan 2012

HAL is a multi-disciplinary open access archive for the deposit and dissemination of scientific research documents, whether they are published or not. The documents may come from teaching and research institutions in France or abroad, or from public or private research centers.
L'archive ouverte pluridisciplinaire HAL, est destinée au dépôt et à la diffusion de documents scientifiques de niveau recherche, publiés ou non, émanant des établissements d'enseignement et de recherche français ou étrangers, des laboratoires publics ou privés. 


\section{Author's Accepted Manuscript}

Multiparticle reaction noise characteristics

Zoran Konkoli

PII:

S0022-5193(10)00624-7

DOI: doi:10.1016/j.jtbi.2010.11.029

Reference: YJTBI 6251

To appear in:

Journal of Theoretical Biology

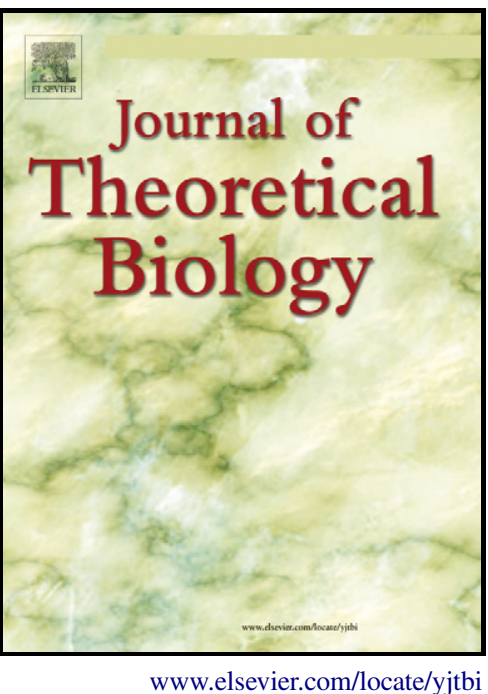

Received date:

22 June 2010

Revised date:

25 October 2010

Accepted date:

23 November 2010

Cite this article as: Zoran Konkoli, Multiparticle reaction noise characteristics, Journal of Theoretical Biology, doi:10.1016/j.jtbi.2010.11.029

This is a PDF file of an unedited manuscript that has been accepted for publication. As a service to our customers we are providing this early version of the manuscript. The manuscript will undergo copyediting, typesetting, and review of the resulting galley proof before it is published in its final citable form. Please note that during the production process errors may be discovered which could affect the content, and all legal disclaimers that apply to the journal pertain. 


\title{
Multiparticle reaction noise characteristics
}

\author{
Zoran Konkoli ${ }^{1, *}$ \\ Bionano Systems Laboratory \\ Department of Microtechnology and Nanoscience - MC2 \\ Chalmers University of Technology \\ Sweden
}

\begin{abstract}
A simple multiparticle reaction model was studied where reactants A react with (a possibly large) stoichiometric coefficient $\mathrm{k}$. Each reaction forms a product molecule $\mathrm{P}$, and every product molecule can split into $\mathrm{k}$ A particles through the back reaction. To study the fluctuations in particle numbers a novel approach has been developed; to be referred to as the Pair approach based Reaction Noise EStimator (PARNES) method. The PARNES method is based on the full Kirkwood superposition approximation implemented at the pair level. Kirkwood's method has been adapted to study stochastic properties of an arbitrary reaction network in a perfectly mixed reaction volume. PARNES works well for large particle numbers. It provides qualitative description when particle numbers are low. The PARNES method can easily augment mean field calculations. Extension of the method beyond the pair approach level is straightforward. Both stationary and non-stationary properties of the model were investigated, and the findings of this work point to two possible scenarios of intracellular noise control. When $\mathrm{k}$ is increased, the fluctuations in the number of product molecules become smaller in a stationary state, and relaxation to a stationary state becomes faster.
\end{abstract}

Keywords: well stirred reaction volume, intracellular noise, PARNES method

\section{Background}

Biochemical reactions in living cells are intrinsically noisy [1]. A large number of theoretical tools have been developed to study intracellular noise [2-5]. Due to low particle numbers, effects of fluctuations are greatly enhanced. In general, living cells adopt two strategies of dealing with noise by either controlling it or using it. On one hand, transcriptional noise needs to be carefully controlled at the single gene level [6], as errors in expression of a gene could have fatal consequences for a cell function [7]. On the other hand, noise can be used to achieve or enhance some function. The trivial example is diffusive transport when molecules are tossed around in the cell interior due to collisions with other molecules. The particle motion generated in such a way is highly irregular but can be an efficient transport mechanism in small volumes [8]. For more complicated examples of constructive use of noise please see [9].

There are many ways to study intracellular noise and a number of related issues need to be understood. Understanding noise control at the single cell level is a highly non-trivial task [10]. On the tissue level, fluctuations are averaged out. There is no randomness in organ functioning since a group of cells act as an ensemble. However, at the single cell level, there is no clear notion of an ensemble that can be averaged over. To facilitate understanding of the noise control at the single cell level, an attempt will be made to describe noise characteristics of multiparticle reactions.

\footnotetext{
*zorank@chalmers.se

${ }^{1}$ www.tabiol.com
}

Preprint submitted to Journal of Theoretical Biology
Truly multiparticle reactions are rare events in a cell interior. The likelihood of many particles meeting at the same time and reacting is negligible $[11,12]$. However, many processes in the cell happen in such a way that a series of reactions with low stoichiometry happen in a short time interval, in a well defined sequence, leaving an impression that they occur simultaneously. Such reactions exhibit a great deal of cooperativity. All reactants have to be present for the successful completion of a reaction chain. The goal will be to understand the noise characterises of such systems.

Complex formation processes are typical examples of a system where cooperative reactions are present. They are ubiquitous in the living cell [13]. The processes are of paramount importance for various forms of intracellular control at all levels, starting from signaling at a cell membrane, through regulation of a gene in the nucleus, and ending with metabolic control. A complex formation process is a combination of random and $\mathrm{co}$ operative assembly steps. [14] A series of binary reactions can proceeded in a well defined order to assemble parts of a complex and all required molecules have to be in place when this happens. This is an example of a cooperative binding. It is also possible that a series of binary reactions occurs in an arbitrary (random) order, but such cases will not be investigated here.

To distinguish experimentally between the two scenarios is hard. Rather elaborate experiments need to be done in order to construct thermodynamic cycles to determine whether a process is cooperative or not. [14] In this work, the focus will be on understanding noise characteristics of cooperative binding and multiparticle reactions. The reactions with low stoichiometry 
numbers have been investigated to a much greater extent.

A conceptually simple model of a multiparticle-like reaction will be studied. All reactants are of the same type $A$ and react cooperatively as $k A \longrightarrow P$, with back reaction $P \longrightarrow k A$ allowed. The multiparticle reaction model used in here is meant as an alias for a sequence of reactions. For example, a complex might be assembled by a series of binary reactions as $A_{1}+A_{i-1} \rightarrow A_{i}$ with $i=2, \ldots, k, A_{1}=A$, and $A_{k}=P$. If the reactions that assemble the complex $A_{k}$ are faster than other processes in the cell, the reactions can be treated as a multiparticle reaction. The advantage of such an approach is that it allows for some elegant modeling. For a given complex formation process which appears to be multiparticle-like, the precise order of the reactions can be ignored. Only the fact that the process is cooperative becomes important. Variable $k$ describes the degree of cooperativity.

There are many process in the cell that are multiparticle-like. For example, parts of Notch signaling, and 30S ribosome assembly processes can be characterized with $k=3$ [14]. There are reactions that need to be described by even larger values of $k$. The assembly of cholesterol-sphingomyelin complexes has $k$ in the range from 3 to 5. [15] The exact value cannot be deduced from experiments. Furthermore, an inspection of the Reactome database [16] which contains information on human pathways indicates that multiparticle-like reactions in the cell are rather frequent. One can find records where the number of inputs $k$ ranges from $k=2$ towards very large values.

Intracellular reactions are organized in pathways. For a given reaction with a set of reactants and a set of products, there are the preceding and the following reactions that provide the reactants and the products. In this study, stochastic properties of whole pathways are not analyzed rigorously but such a situation is taken into consideration nevertheless. Given a part of a reaction pathway that can be represented as a multiparticlelike reaction, it will be assumed that $A$ particles are injected into the system with rate (injection current) $j$. This mimics the situation where reactants $A$ are provided by the preceding reaction. Likewise, molecules $P$ are assumed to spontaneously decay with rate $\gamma$, since products $P$ should be consumed by the reaction that follows. Furthermore, to understand how opening of the system affects noise characteristics, both open and closed systems will be studied in parallel and their behavior compared. The model with $j \neq 0, \gamma \neq 0(j=0, \gamma=0)$ will be referred to as an open (a closed) system.

In the following, the noise characteristics of the model will be analyzed in detail. To do this, a rather generic formalism for performing calculations will be presented.

\section{Results and Discussion}

\subsection{Model description}

The model is defined in equations (1-4). The forward reaction rate is $\lambda$ and the backward (reverse) reaction rate is $\delta$. Symbol $k$ denotes order of the reaction. Particles are assumed to react in clusters of size $k$. A cluster can split into $k A$ particles. It is assumed that particles $A$ are injected with rate $j$.
Once injected the particles move in a closed volume. Symbol $P$ denotes a cluster which is formed by an instance of the forward reaction. Each particle $P$ (cluster) decays with rate $\gamma$. These considerations result in the following set of reactions:

$$
\begin{aligned}
& k A \stackrel{\lambda}{\longrightarrow} P \\
& P \stackrel{\delta}{\longrightarrow} k A \\
& \emptyset \stackrel{j}{\longrightarrow} A \\
& P \stackrel{\gamma}{\longrightarrow} \emptyset
\end{aligned}
$$

It is assumed that the system is studied in the regime where particles mix well. The assumption is correct if the time needed for the reactants to explore the cell is much shorter that the characteristic times scales of the system (the reaction times, the time interval between successive injections, and the time of the decay process). On the other hand if the characteristic time scales are much shorter than the cell exploration time one cannot assume perfect mixing.

For a given molecule, the time $\tau_{m i x}$ to explore the cell grows with the second power of the cell size $L$ as $\tau_{m i x} \sim L^{2} / D$ where $D$ denotes the diffusion constant of the molecule [8]. For example, the time for a protein to diffuse around and explore the cell is roughly $0.1 s$ for E. coli, $10 s$ for Yeast, and $100 s$ for Human Fibroblast where rough estimates for cell volumes are $1 \mu^{3}, 1000 \mu m^{3}$, and $10000 \mu^{3}$ respectively. The examples were taken from [17]. On the other hand, the transition between active and inactive states of a protein happens in the range of $1-100 \mu \mathrm{s}$. These processes are clearly too fast and the perfectly mixing assumption cannot be used to describe them. The time scale of a transcription factor binding to a gene is roughly $1 s$, and the assumption might hold in E. coli. Time to translate a protein is roughly $2 \mathrm{~min}$ and this process could be described with the assumption of perfect mixing.

In here the assumption of perfect mixing is used to simplify the calculations and will not be questioned further. The goal is to investigate how the stochastic properties of the fluctuations in the particle numbers change with varying cooperativity degree $k$.

\subsection{Results}

The quantities we wish to understand are the first (means) and the second (correlations) moments of the particle number distribution $P\left(n_{A}, n_{B}, t\right): \mu_{X}(t)=\left\langle n_{X}\right\rangle$, and $\sigma_{X, Y}(t)=\left\langle\left(n_{X}-\right.\right.$ $\left.\left.\mu_{X}\right)\left(n_{Y}-\mu_{Y}\right)\right\rangle$, where $X, Y \in\{A, P\}$ and symbol $\langle\ldots\rangle$ denotes the average of a fluctuating quantity over distribution $P\left(n_{A}, n_{B}, t\right)$. To study the noise characteristics of the system it is useful to express the standard deviations as

$$
\begin{aligned}
\sigma_{A A}^{2} & =\left(\chi_{A A}-1\right) \mu_{A}^{2}+\mu_{A} \\
\sigma_{P P}^{2} & =\left(\chi_{P P}-1\right) \mu_{P}^{2}+\mu_{P} \\
\sigma_{A P}^{2} & =\left(\chi_{A P}-1\right) \mu_{A} \mu_{P}
\end{aligned}
$$

where $\chi_{A A}$ and $\chi_{P P}$ describe the difference between the (inclusive) particle number distributions and the respective Poisson distributions. For example, a situation when $\chi_{A A}$ and $\chi_{P P}$ are close to one indicates that $A$ and $P$ particle number distributions 
are Poisson like. Furthermore, from (5-7) it is easy to show that $\left\langle n_{A} n_{P}\right\rangle=\chi_{A P}\left\langle n_{A}\right\rangle\left\langle n_{P}\right\rangle$. Accordingly, $\chi_{A P}$ measures to which extent it is possible to approximate $P\left(n_{A}, n_{B}, t\right)$ by the product of univariate particle number distributions for $A$ and $P$ particles. For example, $\chi_{A P} \approx 1$ shows that $P\left(n_{A}, n_{B}, t\right)$ can be factored to a very good approximation and that $\left\langle n_{A} n_{P}\right\rangle \approx\left\langle n_{A}\right\rangle\left\langle n_{P}\right\rangle$.

The particular way of parameterization in (5-7) that uses $\chi_{A A}$, $\chi_{P P}$, and $\chi_{A P}$ is motivated by studies of diffusion controlled reactions in spatially extended systems. (Explained in the section 4.4 in more detail.) In the context of spatially extended systems $\chi_{A A}, \chi_{P P}$, and $\chi_{A P}$ describe correlation functions. For that reason, to avoid coining a new term in this work, symbols $\chi_{A A}, \chi_{P P}$, and $\chi_{A P}$ the will be refereed to as correlation functions, though strictly speaking they are not bound to the $[0,1]$ interval.

Correlation functions $\chi_{A A}$ and $\chi_{P P}$ are related to Mandel's $Q$ parameter [18] used in optics to describe deviations from Poisson distribution. Mandel's parameters are defined as $Q_{A}=$ $\left(\sigma_{A A}^{2}-\langle n\rangle\right) /\langle n\rangle$ and $Q_{P}=\left(\sigma_{P P}^{2}-\langle m\rangle\right) /\langle m\rangle$ and can be easily related to the correlation functions: $\chi_{A A}=1+\frac{Q_{A}}{\langle n\rangle}$ and $\chi_{P P}=1+\frac{Q_{P}}{\langle m\rangle}$.

To compute the moments of interest and perform the analysis of the stochastic properties of the system a suitable pair approximation has been constructed and investigated. In the following the method will be referred to as PARNES, the pair approach based reaction noise estimator. The problem has been mapped to a quantum field theory. The pair approximation has been formulated in the field theory language. The PARNES method amounts to expressing higher order moments of particle number distribution through the pair correlation functions in a particular way as explained in the methods section. This procedure results in a closed set of equations for means $\mu_{A}, \mu_{P}$, and correlation functions $\chi_{A A}, \chi_{P P}$ and $\chi_{A P}$.

The validity of the PARNES method has been carefully examined. The equations have been solved and the results compared to the exact values obtained by diagonalisation of the master equation. For low particle numbers the PARNES approximation provides qualitative agreement with the exact results. For large particle numbers the accuracy of the pair approximation increases tremendously. The deviations from the exact results are uniform in time (remain bound for large times) and the PARNES approach can be used to study stationary states.

The findings that will be discussed hold for the closed and the open system. In a stationary state, the particle number distribution for $A$ particles becomes more Poisson like for large cooperativity degree values $k$. For product molecules $P$ the situation is different. Increase in $k$ leads to larger deviations from the Poisson distribution in such a way that $\sigma_{P P}<\mu_{P}$. The noise in the number of product molecules gets reduced for large reaction orders $k$. Also, the $A P$ pairs become less correlated when $k$ increases. Making the system open increases overall amount of the noise related to the number of $A$ and $P$ particles, and the distribution of the reactant particle number changes significantly.

At the level of the PARNES approximation, the value of cooperativity degree $k$ has a significant impact on how fast the system approaches a stationary state. (The analysis has been performed for closed systems only but in the light of the above discussion it is expected to hold for open systems as well.) For large reaction orders that involve clusters with big sizes the approach to a stationary state is much faster. Interestingly, this effect gets suppressed for small $\delta / \lambda$ ratios (forward reaction dominates). In general, when ratio $\delta / \lambda$ is increased system approaches a stationary state faster. This is a non trivial result in a sense that it involves ratio of the reaction rate constants for the forward and the backward reaction and not absolute values of the reaction rates.

\section{Conclusions}

The emphasis of the work was on understanding the noise characteristics of multiparticle-like reactions. The relatively simple reaction model was investigated. To study the stochastic properties of the system a novel method of computation was constructed and referred to as PARNES. The method is based on the idea of the pair approximation used in various many body theories. For example, the pair approach is frequently used in chemical kinetics studies of spatially extended diffusion controlled reactions. The original technique was adapted to describe the chemical system in a well mixed reaction volume. In technical terms this is somewhat non-trivial task. The validity of the PARNES method has been carefully investigated by comparing with the exact master equation diagonalisation method. The PARNES approach describes the system qualitatively at low copy numbers. For large particle numbers the accuracy of the method greatly improves.

The approach suggested here is generic and can be easily applied to study more complicated cases where an exact solution of the problem is not possible, for example, where exact diagonalisation of the master equations is computationally too demanding. This will likely happen when a system level analysis needs to be performed for a large pathway. The method naturally extends the mean field equations to take into account effects of noise at the lowest order. The parameters used to formulate the mean field model can be reused. There is no need to introduce new parameters. In addition, the approach provides a way for (almost) analytical understanding of a system.

The reaction studied has few interesting properties that might be relevant for understanding the noise characteristics of multiparticle reactions. Increase in the cooperativity degree $k$ leads to the better noise control of the product molecules at a stationary state. The reactant particle number becomes Poisson distributed for large reaction orders. This finding holds irrespectively of whether open or closed systems are considered. An open system is expected to be more noisy, but the dependence of the noise characteristics on the value of $k$ is roughly the same. This shows that it is possible to study a reaction in isolation and still be able to draw useful conclusions about the situation when the reaction is part of a pathway.

Two types of processes are present in the cell. On one hand, many processes are driven and occur at a stationary state with constant inflow and outflow of particles. On the other hand, there are also processes that are strongly time dependent and 
should be modeled in a fundamentally different way. [10] Typical examples are signaling events that have clear beginning and end in time (e.g. when ligand binds, and last molecule in a cascade is formed), various temporal programs[3], or biochemical clocks (e.g. circadian) where periodic altering of particle number brings persistent change that the system needs to respond to. The findings of this work might bear some relevance on understanding such processes. For example, approach to the equilibrium is faster for large $k$ values. (In that respect only closed system was studied. Since making the system open did not alter its stationary state characteristics the same is expected to hold for an equilibration process.) The fact that the system reaches a stationary state faster for large $k$ values is a non trivial statement. The statement refers to the noise characteristics of the whole system, not only being restricted on average particle numbers.

Biophysical and biochemical constraints are the most likely reason for the existence of complex formation since macromolecular complexes frequently involve catalytic units. However, it is possible that some complex formation reactions play a crucial role in controlling intracellular noise. [10] This work points to two particular mechanism of a noise control. Occurrence of a multiparticle-like reaction in a pathway might signify two things. The noise reduction unit might be in place to reduce fluctuations in the number of product molecules. Another possibility is that the module stabilizes the desired noise characteristics by enhancing the approach to the local stationary state.

The $k A \rightarrow P$ reaction (back reaction forbidden) has been studied in the infinite volume limit for arbitrary dimension of the system $d$ [11]. The upper critical dimension of the system is equal to $d_{c}=2 /(k-1)$. For $d>d_{c}$ mean field kinetics can be used to describe the system, while for $d \leq d_{c}$ the system exhibits anomalous kinetics and mean field equations cannot be used. The critical dimension gets lower as one increases the order of the reaction $k$. For large $k$ values it is less likely for large clusters of $A$ particles to meet, reactions are infrequent and cannot disturb the well mixed state. Presence of the anomalous kinetics implies large degree of spatiotemporal fluctuation. In such a way large $k$ values lead to the improved noise control. This suppresses occurrence of anomalous kinetics which occurs only in relatively low dimensions. The analysis performed in this work demonstrated that behaviors of the spatially extended and well mixed systems are similar in a sense that large $\mathrm{k}$ values lead to the improved noise control.

There are a number of ways how the present approach could be extended. The intuitive assumption that cooperative processes can be approximated as multiparticle reactions should be checked formally. For example, it would be interesting to see whether the same results hold when mixing is not perfect. Also, most of the discussion focused on the situation when the system is prepared in a pure state. The analysis in this work could be easily extended to other types of initial conditions that involve groups (ensemble) of cells with predefined noise characteristics. Furthermore, it would be interesting to try do identify specific characteristics that set random and cooperative binding apart.

\section{Methods}

\subsection{Master equation}

The noise we wish to investigate is related to the discreetness of the particle number. In order to describe the system one needs to define the structure of its configuration space. The configurations of the system will be specified by counting the number of particles in the system. The positions of the particles will not be followed. The particle positions are irrelevant due to the perfect mixing assumption. A configuration of the system is given by $c=\left(n_{A}, n_{P}\right)$ where $n_{A}$ and $n_{P}$ denote number of $\mathrm{A}$ and $\mathrm{P}$ particles respectively.

The ensemble average of any observable (function of variables $n_{A}$ and $n_{P}$ ) is given by

$$
\left\langle f\left(n_{A}, n_{P}\right)\right\rangle=\sum_{n_{A}, n_{P}} f\left(n_{A}, n_{P}\right) P\left(n_{A}, n_{P}, t\right)
$$

where $P\left(n_{A}, n_{P}, t\right)$ denotes the probability of finding the system in a configuration $\left(n_{A}, n_{P}\right)$. To make the notation for particle numbers more compact, definitions $n \equiv n_{A}$ and $m \equiv n_{P}$ will be used.

The problem at hand can be described by the master equation that describes changes of the occupancy probabilities for each state

$$
\frac{\partial}{\partial t} P(n, m, t)=\lambda \mathcal{J}_{f}+\delta \mathcal{J}_{b}+j \mathcal{J}_{\text {in }}-\gamma \mathcal{J}_{\text {out }}
$$

In master equation (9) contributions for the forward and the back reactions have been separated, together with the source, and the sink terms. The forward reaction contribution is described by

$$
\mathcal{J}_{f}=\left(\begin{array}{c}
n+k \\
k
\end{array}\right) P(n+k, m-1, t)-\left(\begin{array}{l}
n \\
k
\end{array}\right) P(n, m, t)
$$

and the backward reaction as

$$
\mathcal{J}_{b}=(m+1) P(n-k, m+1, t)-m P(n, m, t)
$$

The binomial coefficients describe how many clusters of $k$ particles are in the system at any time instant. The injection of particles is described with

$$
\mathcal{J}_{\text {in }}=P(n-1, m, t)-P(n, m, t)
$$

and the decay as

$$
\mathcal{J}_{\text {out }}=(m+1) P(n, m+1, t)-m P(n, m, t)
$$

To evaluate averages over $P\left(n_{A}, n_{B}, t\right)$ as in (8) one needs to solve master equation (9) and then perform the sum. This route is rather complicated in practice. To facilitate the analytical understanding of the problem another method will be used. The equations of motion will be derived for the observables of interest and the equations of motion will be solved directly using the pair approximation ansatz adapted to the perfect mixing setup studied in here.

The observables of interest are factorial moments defined as

$$
\begin{aligned}
\rho(x, y, t) \equiv & \langle n(n-1)(n-2) \ldots(n-x+1) \times \\
& m(m-1)(m-2) \ldots(m-y+1)\rangle
\end{aligned}
$$


To derive the equations of motion in the straight forward way, one should simply take the time derivative of equation (8), with observable defined in (14), and doing all the necessary technical steps. However, such route would be rather tedious.

To find the equations of motion another approach will be followed. The problem will be mapped first on to the equivalent quantum field theory problem, and the equations of motion will be derived using the field theory formalism. In this context the field theory provides a powerful book-keeping device which facilitates calculations. In the following sub-section some technical preliminaries will be discussion.

\subsection{Notation preliminaries}

The problem is mapped onto a quantum field theory by using standard techniques. Please see [19] for a recent review. In the following only essential steps of the calculation will be listed.

Dynamics of the system is conveniently described using the generating function

$$
|\psi(t)\rangle=\sum_{n, m} P(n, m, t)\left(\hat{a}^{\dagger}\right)^{n}\left(\hat{p}^{\dagger}\right)^{m}|0\rangle
$$

where $\hat{a}^{\dagger}$ and $\hat{p}^{\dagger}$ denote the creation operators for the reactant and the product molecules respectively. The operators without the dagger are the corresponding annihilation operators, and $|0\rangle$ denotes the vacuum state. In the vacuum state there are no particles to annihilate leading to

$$
\hat{a}|0\rangle=0, \quad \hat{p}|0\rangle=0
$$

However, particles can be created and the state of the system in a configuration $c=(n, m)$ can be generated by creating $n A$ and $m P$ particles as

$$
|n, m\rangle=\left(\hat{a}^{\dagger}\right)^{n}\left(\hat{p}^{\dagger}\right)^{m}|0\rangle
$$

Following commutation rules are valid for the operators

$$
\left[\hat{a}, \hat{a}^{\dagger}\right]=1 \quad\left[\hat{p}, \hat{p}^{\dagger}\right]=1
$$

and all other commutators are zero. The commutator symbol is defined in the standard way as $[\hat{X}, \hat{Y}]=\hat{X} \hat{Y}-\hat{Y} \hat{X}$. By using (18) it is possible to see that applying the particle number operators $\hat{n}_{A} \equiv \hat{a}^{\dagger} \hat{a}$ and $\hat{n}_{P} \equiv \hat{p}^{\dagger} \hat{p}$ on the vector representing the state $c$ gives $\hat{n}_{A}|n, m\rangle=n|n, m\rangle$ and $\hat{n}_{P}|n, m\rangle=m|n, m\rangle$ as expected. For example, $\hat{n}_{A}|1,0\rangle=\hat{a}^{\dagger} \hat{a} \hat{a}^{\dagger}|0\rangle$, which upon using (18) becomes $\left(\hat{a}^{\dagger}+\hat{a}^{\dagger} \hat{a}^{\dagger} \hat{a}\right)|0\rangle$, and finally through (16) gives $\hat{a}^{\dagger}|0\rangle$, or equivalently $|1,0\rangle$. All these steps result in $n_{A}|1,0\rangle=1|1,0\rangle$. Actually, it is possible to show that $f\left(\hat{a}^{\dagger} \hat{a}, \hat{p}^{\dagger} \hat{p}\right)|n, m\rangle=f(n, m)|n, m\rangle$ where $f$ is any bivariate function of integer arguments.

The generating function is a linear combination of all possible states of the system weighted by the occupancy probabilities. Accordingly, the master equation (9) can be represented as the equation which involves the generating function solely as

$$
\frac{\partial}{\partial t}|\psi(t)\rangle=-\hat{H}|\psi(t)\rangle
$$

where $\hat{H}$ should be a linear operator since the master equation is linear. The requirement of the equivalence between (9) and
(19) defines the form of the Hamiltonian operator $\hat{H}$, which in the case of the particular model studied is given by

$$
\hat{H}=\left[\left(\hat{a}^{\dagger}\right)^{k}-\hat{p}^{\dagger}\right]\left(\frac{\lambda}{k !} \hat{a}^{k}-\delta \hat{p}\right)+j\left(1-\hat{a}^{\dagger}\right)+\gamma\left(\hat{p}^{\dagger}-1\right) \hat{p}
$$

It requires some work to see this. Since the calculation is rather long it will not be reproduced in here. However, curious reader can insert (15) into equation (19) to obtain the agreement.

For example, applying the source term in the Hamiltonian onto the generating function gives

$$
j\left(1-\hat{a}^{\dagger}\right)|\psi(t)\rangle=j \sum_{n, m}(P(n, m, t)-P(n-1, m, t))|n, m\rangle
$$

The term in the parentheses is exactly the source term (12) in the master equation. The term can be extracted by acting with $\langle n, m|=\langle 0| \hat{a}^{n} \hat{p}^{m}$ on (19) or (21), and using orthogonality condition $\left\langle n, m \mid n^{\prime}, m^{\prime}\right\rangle \propto \delta_{n, n^{\prime}} \delta_{m, m^{\prime}}$ where $\delta$ denote the Kronecker delta function. The orthogonality condition follows from (16) and (18).

In the language of the field theory an average of any observable can be calculated as $\langle f(n, m)\rangle=\langle\hat{f}\rangle$ where $\hat{f}=f\left(\hat{a}^{\dagger} \hat{a}, \hat{p}^{\dagger} \hat{p}\right)$ denotes the quantum field theory operator that corresponds to an observable we wish to compute. Once the operator is constructed quantum field averages are computed as

$$
\langle\hat{f}\rangle=\langle 1|\hat{f}| \psi(t)\rangle
$$

Symbol $<1 \mid$ stands for the left eigenvector of the annihilation operators, and is defined as

$$
|1\rangle=\exp \left(\hat{a}^{\dagger}+\hat{p}^{\dagger}\right)|0\rangle
$$

where $\langle 1| \hat{a}^{\dagger}=\langle 1| \hat{p}^{\dagger}=\langle 1|$. Requirement $\langle 1 \mid \psi(t)\rangle=1$ expresses the fact that $P(n, m, t)$ is normalized to one.

Equations of motion for any observable can be derived by taking the time derivative of (22) which gives

$$
\frac{\partial}{\partial t}\langle\hat{f}\rangle=-\langle[\hat{f}, \hat{H}]\rangle
$$

In the field theoretic formalism the factorial moments can be computed by realizing that

$$
\rho(x, y, t)=\left\langle\hat{a}^{x} \hat{p}^{y}\right\rangle
$$

and using (24). Please note that factorial moments become density functions in the jargon of quantum field theory. In the following subsection equation (24) will be used to find the equations of motion for density functions.

\subsection{Deriving equations of motion}

Using equation (24) with $\hat{f} \in\{\hat{a}, \hat{p}\}$ gives the equations of motion for the average particle numbers

$$
\begin{aligned}
\frac{\partial}{\partial t}\langle\hat{a}\rangle & =-\frac{\lambda}{(k-1) !}\left\langle\hat{a}^{k}\right\rangle+\delta k\langle\hat{p}\rangle+j \\
\frac{\partial}{\partial t}\langle\hat{p}\rangle & =\frac{\lambda}{k !}\left\langle\hat{a}^{k}\right\rangle-\delta\langle\hat{p}\rangle-\gamma\langle\hat{p}\rangle
\end{aligned}
$$


Also, using equation (24) with $\hat{f} \in\left\{\hat{a}^{2}, \hat{p}^{2}, \hat{a} \hat{p}\right\}$ gives the desired equations for quantities related to the standard deviations

$$
\begin{aligned}
\frac{\partial}{\partial t}\left\langle\hat{a}^{2}\right\rangle & =\left\langle[k(k-1)+2 k \hat{a}]\left(-\frac{\lambda}{k !} \hat{a}^{k}+\delta \hat{p}\right)+2 j \hat{a}\right\rangle \\
\frac{\partial}{\partial t}\left\langle\hat{p}^{2}\right\rangle & =\left\langle 2 \hat{p}\left(\frac{\lambda}{k !} \hat{a}^{k}-\delta \hat{p}\right)-2 \gamma \hat{p}^{2}\right\rangle \\
\frac{\partial}{\partial t}\langle a p\rangle & =\left\langle[k \hat{p}-\hat{a}]\left(-\frac{\lambda}{k !} \hat{a}^{k}+\delta \hat{p}\right)+j \hat{p}-\gamma \hat{a} \hat{p}\right\rangle
\end{aligned}
$$

Please note that equations above involve the terms which have not been discussed yet. For example, equation for $\left\langle\hat{a}^{2}\right\rangle$ depends on $\left\langle\hat{a}^{k}\right\rangle$, equation for $\left\langle\hat{p}^{2}\right\rangle$ depends on $\left\langle\hat{p} \hat{a}^{k}\right\rangle$ etc. The emergence of an infinite hierarchy of equations is an expected behavior. Equations (28-30) are just the beginning of the hierarchy. To solve the problem one needs to cut the hierarchy in some way. To close the equations the approaches discussed in [20] and [21] will be used and adapted to describe a well mixed case.

\subsection{The pair approximation}

The general discussion of how to decouple similar hierarchies of equations can be found in [20]. The paper [21] showed how to implement these ideas for spatially extended system, where multiple occupancy of lattice sites is allowed. It is straight forward to modify the techniques presented in [21] to describe a perfectly mixed reaction volume. A single lattice site (with a multiple occupancy allowed) is equivalent to a perfectly mixed reaction volume studied in here. These ideas are expressed mathematically as follows.

It is convenient to introduce pair correlation functions for $A A$, $P P$, and $A P$ pairs as

$$
\begin{aligned}
& \langle\hat{a} \hat{a}\rangle \equiv\langle\hat{a}\rangle^{2} \chi_{A A}(t) \\
& \langle\hat{p} \hat{p}\rangle \equiv\langle p\rangle^{2} \chi_{P P}(t) \\
& \langle\hat{a} \hat{p}\rangle \equiv\langle\hat{a}\rangle\langle\hat{p}\rangle_{A P}(t)
\end{aligned}
$$

The issue is how to express the averages of larger powers of annihilation operators using pair correlation functions defined above. Inspired by discussion in [20] and [21], following approximation will be used to solve the problem

$$
\left\langle\hat{a}^{x} \hat{p}^{y}\right\rangle \approx\langle\hat{a}\rangle^{x}\left\langle\hat{p} y^{y} \chi_{A A}(t)^{\left(\begin{array}{l}
x \\
2
\end{array}\right)} \chi_{P P}(t)^{\left(\begin{array}{l}
y \\
2
\end{array}\right)} \chi_{A P}(t)^{x y}\right.
$$

where $x$ and $y$ are integers larger or equal to zero. Please note that the exponents on the correlation functions count number of $A A, P P$, and $A P$ pairs in the system with $x A$ and $y P$ particles. Equation (34) represents an instance of Kirkwood's superposition approximation [20,21], more specifically of the full Kirkwood's pair approximation. This approximation is central for workings of the PARNES method. The approximation completely decouples the equations of motion.

Using (34) equations (26-27) become

$$
\begin{aligned}
& \frac{\partial}{\partial t} a \approx-\frac{\lambda}{(k-1) !} a^{k} \chi_{A A}^{\frac{1}{2} k(k-1)}+\delta k p+j \\
& \frac{\partial}{\partial t} p \approx \frac{\lambda}{k !} a^{k} \chi_{A A}^{\frac{1}{2} k(k-1)}-(\delta+\gamma) p
\end{aligned}
$$

where notation $a \equiv\langle\hat{a}\rangle$ and $p \equiv\langle\hat{p}\rangle$ was used. In a similar way, after using the pair approximation, equations (28-30) turn into

$$
\begin{aligned}
\frac{\partial}{\partial t} \chi_{A A} \approx & -\frac{\lambda}{(k-2) !} a^{k-2} \chi_{A A}^{\frac{1}{2} k(k-1)}+\delta k(k-1) \frac{p}{a^{2}} \\
& +2 k \delta \frac{p}{a}\left(\chi_{A P}-\chi_{A A}\right) \\
& -\frac{2 \lambda}{(k-1) !} a^{k-1} \chi_{A A}^{\frac{1}{2} k(k-1)}\left(\chi_{A A}^{k}-\chi_{A A}\right)+2 j \\
\frac{\partial}{\partial t} \chi_{P P} \approx & \frac{2 \lambda}{k !} \frac{a^{k}}{p} \chi_{A A}^{\frac{1}{2} k(k-1)}\left(\chi_{A P}^{k}-\chi_{P P}\right)-2 \gamma \chi_{P P} \\
\frac{\partial}{\partial t} \chi_{A P} \approx & -\frac{\lambda}{(k-1) !} a^{k-1} \chi_{A A}^{\frac{1}{2} k(k-1)}\left(\chi_{A P}^{k}-\chi_{A P}\right) \\
& +k \delta \frac{p}{a}\left(\chi_{P P}-\chi_{A P}\right) \\
& +\frac{\lambda}{k !} \frac{a^{k}}{p} \chi_{A A}^{\frac{1}{2} k(k-1)}\left(\chi_{A A}^{k}-\chi_{A P}\right)+\frac{j}{a}-\chi_{A P}
\end{aligned}
$$

\subsection{Relation to other methods}

Before discussing applications of the PARNES method, it will be briefly compared to the other approaches that work in the same setup where perfect mixing is assumed and particles are counted (particle position is not traced). One strives to develop proper closure method for equations of motion. Description of the methods can be found in [3-5]. The methods described in [3-5] work essentially on the master equation level where master equation is manipulated to arrive at the closed set of expressions.

The PARNES method is essentially an ansatz for particle number distribution being expressed as a relationship between moments. The ansatz is expressed through Eq. (34). Since Eq. (25) holds for any value of $x$ and $y$ (larger than or equal zero) one can deduce actual form of the particle number distribution used by comparing (25) with (34). This will not be done in here as it is a non-trivial problem in mathematical statistics (the inverse moment problem).

There are both advantages and disadvantages of working with the ansatz setup. The advantages will be discussed first. For example, van Kampen's method implicitly assumes that one can perform the Taylor like (additive) expansion in the noise effects. As an artefact of that, in van Kampen's method, the first moments are decoupled from second moments. The equations for averages are not improved. In contrast to this, no such assumptions are made in the construction of the PARNES method and, accordingly, first moments couple to second moments. It has not been tested rigorously, but very likely the PARNES method effectively re-sums a part of the van Kampen's series. Furthermore, one is not restricted to work in proximity of a stationary state (e.g. as in the method discussed in [3]).

Last but not least, the PARNES method is open-ended since one can easily go beyond the pair approximation level should a need occur. It is straight forward to generalize the methods discussed for spatially extended systems in [20] and [21] to account for higher order effects in a perfect mixing setup.

However, the advantages discussed above bring an obvious disadvantage. The only way to justify the pair ansatz is to compare results of the PARNES method with an exact solution to a 
problem under investigation. Such calculations have been done in the context of the Ising model, and are cited in [20]. It was shown that Kirkwood's superposition approximation can provide accurate results. This is in agreement with findings of this work.

\subsection{Analysis of closed system $(j=0, \gamma=0)$}

Once the method of solving the problem has been presented we proceed by discussing actual behavior of the system under investigation. For the closed system two conservation laws can be extracted from the equations of motion. Both laws arise from the fact that $n+k m$ and $(n+k m)^{2}$ (or any other function of $n+k m$ ) are conserved:

$$
\begin{aligned}
& \frac{\partial}{\partial t}[a+k p]=0 \\
& \frac{\partial}{\partial t}\left[a^{2} \chi_{A A}+a+k^{2}\left(p^{2} \chi_{P P}+p\right)+2 k a p \chi_{A P}\right]=0
\end{aligned}
$$

One can show that equations above are valid by performing straight forward evaluation of the time derivatives by using (3539 ) and showing that the time derivatives evaluate to zero.

\subsubsection{Accuracy of the pair approximation}

Figures 6.1-6.2 depict the error of the PARNES method as the function of time. The error for a quantity $q \in$ $\left\{a, p, \chi_{A A}, \chi_{P P}, \chi_{A P}\right\}$ is defined as

$$
\epsilon(q, t)=\frac{|q(t)-\hat{q}(t)|}{\hat{q}(t)}
$$

where $\hat{q}(t)$ denotes the exact and $q(t)$ the approximate value of the quantity at any time instant $t$. The exact values were obtained by using (8) after the straight forward diagonalisation of master equation (9). The diagonalisation procedure is computationally rather demanding for large particle numbers and errors were computed for the case with relatively small particle numbers.

Instead of plotting error $\epsilon(q)$ for each quantity $q$ only the maximum error, $\max _{q}\{\epsilon(q)\}$, is depicted. From figure 6.1 one can see that the maximum error increases for large $k$ values. Also, both figures (and especially figure 6.2) show that the maximum error gets reduced for large particle numbers. Already for relatively low particle numbers there is a very good agreement with the exact results. Please note that the error is uniform in time in all cases. This has to do with the (non-trivial) fact that the observables calculated with the PARNES method saturate to constant values for large times. Accordingly, for large copy numbers, and not too high $k$ values, the PARNES approach can quantitatively describe stationary states.

Very likely, the pair approximation is well suited for describing a situation where all correlation functions are close to one. This in turn implies that the standard deviations have a Poisson like behavior with $\sigma_{A A} \approx \mu_{A}$ and $\sigma_{P P} \approx \mu_{P}$. It also implies that $A P$ pairs are uncorrelated $\sigma_{A P} \approx \mu_{A} \mu_{P}$. This is probably the reason why the pair approximation cannot describe low copy numbers. For the pure states that were studied here the initial particle distribution strongly differs from a Poisson distribution. As the particle numbers are increased this problem becomes less pronounced.

\subsubsection{Equilibrium state}

Once the validity of the pair approach has been critically examined the discussion will focus on the properties of the reaction system. In the case of a closed system a stationary state is also an equilibrium state. The equations that describe the stationary state are obtained by zeroing the time derivatives on the left hand side of equations (35-39). Equations (35) and (36) give following condition

$$
\frac{\lambda}{k !} a_{*}^{k}\left[\chi_{A A}^{*}\right]^{k(k-1) / 2}=\delta p_{*}
$$

where $a_{*}=\lim _{t \rightarrow \infty} a(t), p_{*}=\lim _{t \rightarrow \infty} p(t)$, and $\chi_{A A}^{*}=$ $\lim _{t \rightarrow \infty} \chi_{A A}(t)$. Equations (37-39) result in

$$
\begin{aligned}
& {\left[\chi_{A P}^{*}\right]^{k}=\chi_{P P}^{*}} \\
& {\left[\chi_{A A}^{*}\right]^{k}=\chi_{A P}^{*}}
\end{aligned}
$$

where $\chi_{P P}^{*}=\lim _{t \rightarrow \infty} \chi_{P P}(t)$ and $\chi_{A P}^{*}=\lim _{t \rightarrow \infty} \chi_{A P}(t)$. Interestingly, the three equations result in two conditions only.

There is a set of stationary states parameterized by two free parameters. Any tuple of the form $\left(a_{*}, p_{*}, \chi_{A A}^{*}, \chi_{P P}^{*}, \chi_{A P}^{*}\right)$ can be a stationary state as long as equations (43-44) are satisfied. There are five variables with three equations indicating that two variables are free. The free variables can be found from the fact that there are two conservation laws. Using (40) and (41) results in two additional equations

$$
\begin{aligned}
& a_{*}+k p_{*}=\alpha_{0} \\
& a_{*}^{2} \chi_{A A}^{*}+a_{*}+k^{2}\left(p_{*}^{2} \chi_{P P}^{*}+p_{*}\right) \\
& \quad+2 k a_{*} p_{*} \chi_{A P}^{*}=\beta_{0}
\end{aligned}
$$

where $\alpha_{0}$ and $\beta_{0}$ are constants depending only on the initial condition values;

$$
\begin{aligned}
\alpha_{0}= & a_{0}+k p_{0} \\
\beta_{0}= & a_{0}^{2} \chi_{A A}^{0}+a_{0}+k^{2}\left(p_{0}^{2} \chi_{P P}^{0}+p_{0}\right) \\
& +2 k a_{0} p_{0} \chi_{A P}^{0}
\end{aligned}
$$

Variables $\alpha_{0}$ and $\beta_{0}$ can be chosen as the two free parameters. The system of equations (43-47) is complete but will not be analyzed in here. The more detailed analysis of the equilibrium state is left for a future work.

\subsubsection{Non equilibrium properties of the reaction}

Many processes in the cell are non-equilibrium like, and in the following it will be investigated how fast the system equilibrates. To perform such analysis the equations of motion will be linearized in the vicinity of the stationary state. It is convenient to introduce small deviations from a stationary state solution, $q_{v}^{*}$, for all quantities of interest (average particle numbers and standard deviations) as $q_{\nu}(t) \approx q_{v}^{*}+u_{\nu}(t)$. Using the new variables $u_{v}$ with $v=1, \ldots, 5$, and assuming that the derivations are small, one can obtain the linearized equations of motion

$$
\frac{d \mathbf{u}}{d \tau}=-\mathbb{M} \mathbf{u}
$$

where all variables have been arranged into the column vector $\mathbf{u}^{\top}=\left(u_{1}, \ldots, u_{5}\right)$. The analytical form of the $\mathbb{M}$ matrix can be 
found but it will not be shown in here. In order to simplify the numerical work the time has been scaled as $\tau=\lambda t$.

The matrix $\mathbb{M}$ has been computed numerically for the pure initial states with known values for $a_{0}$ and $p_{0}$. The matrix has been diagonalised numerically which gave the eigenvalues according to the equation

$$
\mathbb{M} \mathbf{l}_{v}=\omega_{v} \mathbf{l}_{v}
$$

with $v=1, \ldots, 5$.

For convenience it will be assumed that the eigenvalues are sorted so that $\omega_{1}<\omega_{2}<\ldots<\omega_{5}$. The first two eigenvalues are always zero. This has to do with the fact that the stationary states are parameterized by two free parameters $\alpha_{0}$ and $\beta_{0}$. Out of the remaining three non zero eigenvalues only the eigenvalue with the smallest real part will be of interest. This eigenvalue controls rapidity of the equilibration process. Results of the numerical analysis are shown in figures 6.6 and 6.7.

\subsection{Analysis of open system $(j \neq 0, \gamma \neq 0)$}

Figures 6.8-6.10 show the behavior of the correlation functions for the open system. Please see figures 6.3-6.5 depicting correlation functions for the closed system for a comparison. The inspection of figures 6.3 and 6.8 reveals that, in contrast to the closed system, increase in $k$ reduces the fluctuations in the reactant particle number. In both (open and closed) cases the process of increasing $k$ makes the particle number distributions more Poisson like. For the open (closed) systems studied in the figures the correlation functions approach one from above (below) but this is dependent on the exact value of $j$ (and other parameters). Also, one can see that if the system is open the overall level of noise increases, which is not surprising.

The pair correlation function for $P$ particles exhibits similar behavior for the open and the closed systems. This can be seen by comparing figures 6.4 and 6.9 . Increase in $k$ reduces the amount of fluctuations in the number of $P$ particles. Again, if the system is open the overall amount of noise increases; curves in Fig. 6.9 are closer to one than corresponding curves in Fig. 6.4.

Comparison of figures 6.5 and 6.10 reveals an interesting behavior of the $A P$-correlation function. Opening the system reduces the amount of the correlation between the $A P$ pairs. If the system is closed, one expects that $A$ and $P$ particle numbers should be highly correlated. A spontaneous fluctuation leading to a large number of $A$ particles implies that the number of $P$ particles is low, and vice versa. This has to do with the presence of the conservation laws discussed previously for the closed system where the decay and the inflow of reactants are not allowed. Opening the system weakens such behavior. In both (open and closed) cases increase in $k$ makes the $A P$ pairs less correlated as the correlation function approaches one. However, for the particular value $j$ used the approach occurs in two different ways, from above (below) for an open (a closed) system. Accordingly, when $k$ is large, one has $\langle n m\rangle>\langle n\rangle\langle m\rangle$ $(\langle n m\rangle\langle\langle n\rangle\langle m\rangle)$ for an open (a closed) system.

\section{Acknowledgements}

The author would like to acknowledge financial support from Chalmers Biocenter and MC2 grant for strategic development. Illuminating discussions with Vincent Danos regarding macromolecular complex formation are greatly appreciated. The author wishes to thank Aldo Jesorka for useful discussions which greatly improved the readability of the manuscript.

\section{Figures}

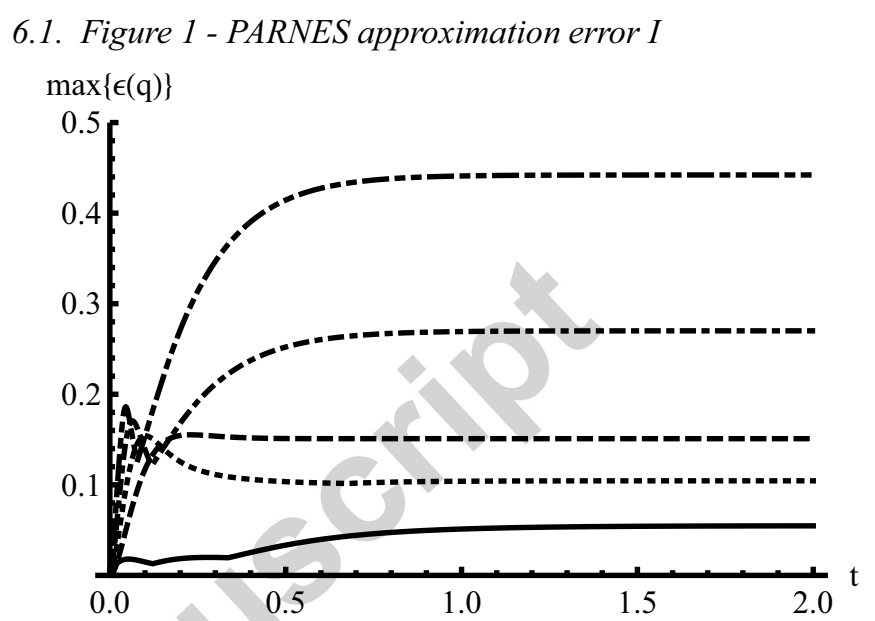

The maximum relative error plot showing the difference between the exact results and the PARNES approximation; $q \in\left\{a, p, \chi_{A A}, \chi_{P P}, \chi_{A P}\right\}$. Time is expressed in units of $1 / \lambda$ (valid for all plots that depict time dependence). All plots were obtained with $\delta / \lambda=1, a_{0}=p_{0}=2$ and $k=2$ (full line), $k=3$ (dashed line), $k=4$ (dotted), $k=5$ (dot-dash), and $k=6$ (dot-dot-dash). The maximum error gets larger for large $k$ values and can be uniformly bound in $t$.

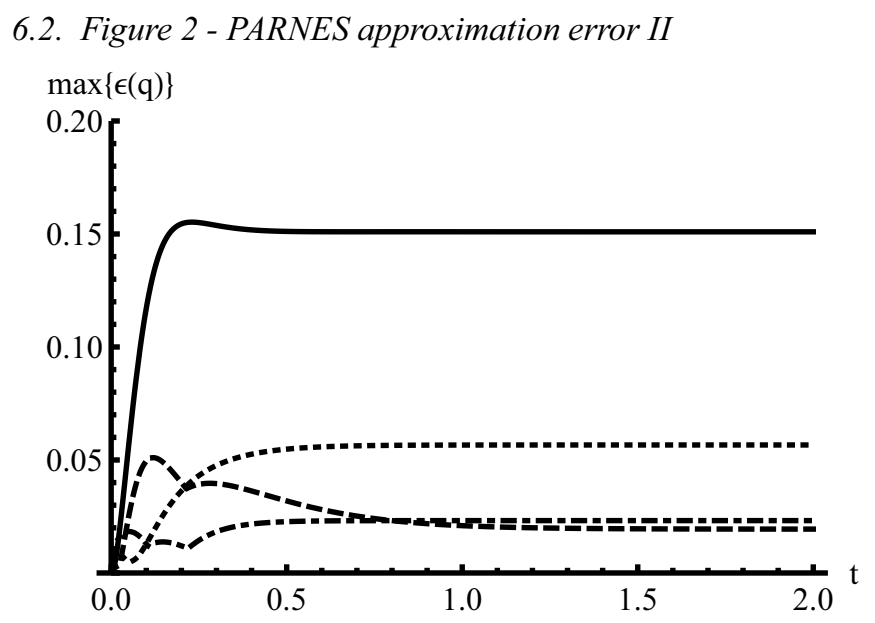

The maximum relative error plot showing the difference between the exact results and the PARNES method; $q \in$ $\left\{a, p, \chi_{A A}, \chi_{P P}, \chi_{A P}\right\}$. Plots were obtained with $\delta / \lambda=1, k=3$, and $a_{0}=p_{0}=2$ (full line), $a_{0}=p_{0}=3$ (dashed line), $a_{0}=p_{0}=4$ (dotted), $a_{0}=p_{0}=5$ (dot-dash). The maximum error gets smaller for large particle numbers and the errors can be uniformly bound in $t$. 
6.3. Figure 3 - AA pairs correlation function

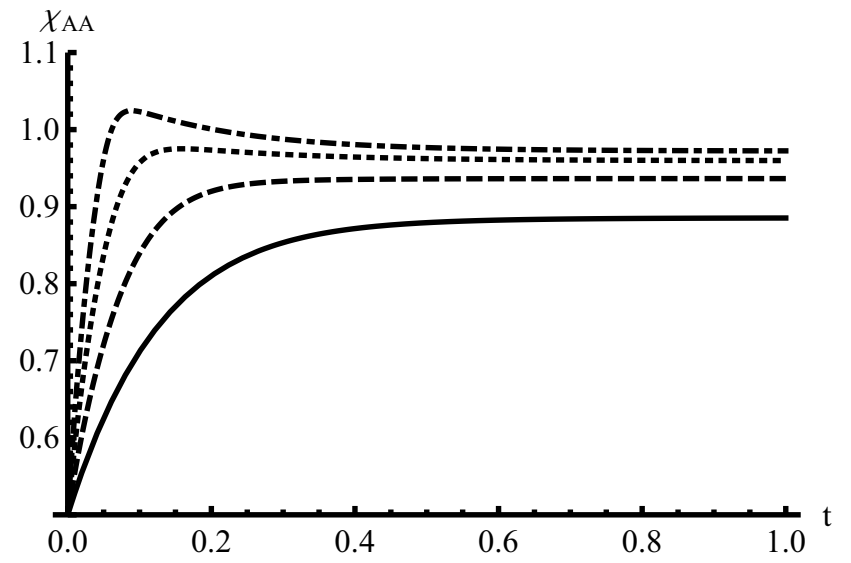

The plots were generated for $\delta / \lambda=1, a_{0}=p_{0}=2$ and $k=2$ (full line), 3 (dashed line), 4 (dotted line), and 5 (dot-dashed line). For large $k$ values the equilibrium distribution becomes more Poisson like (the particle number distribution becomes wider).

\subsection{Figure 4 - PP pairs correlation function}

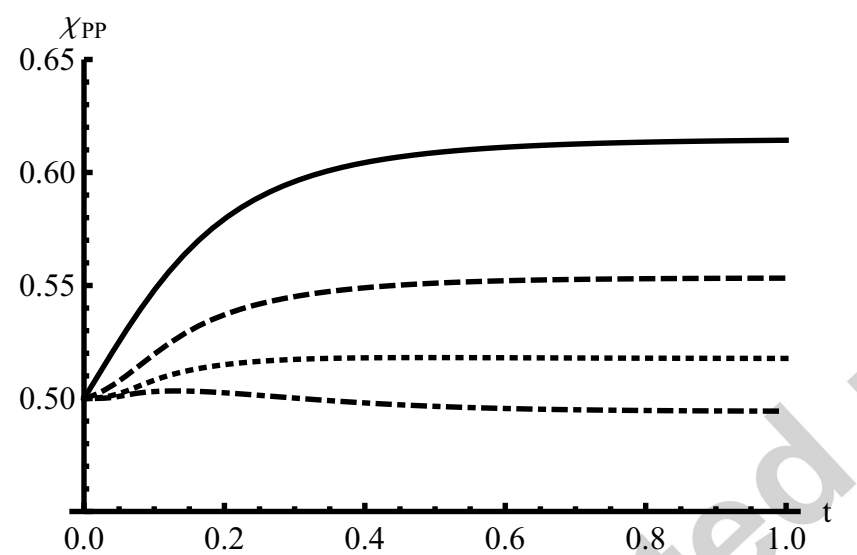

The plots were generated in the same way as for figure 6.3. In contrast to the $A A$ pair correlation function, the $P P$ pair correlation function describes larger deviation from the Poisson distribution for large $k$ values. The amount of noise is reduced for large $k$ values since the particle number distribution becomes increasingly sub-Poissonian (more narrow).

\subsection{Figure 5 - AP pairs correlation function}

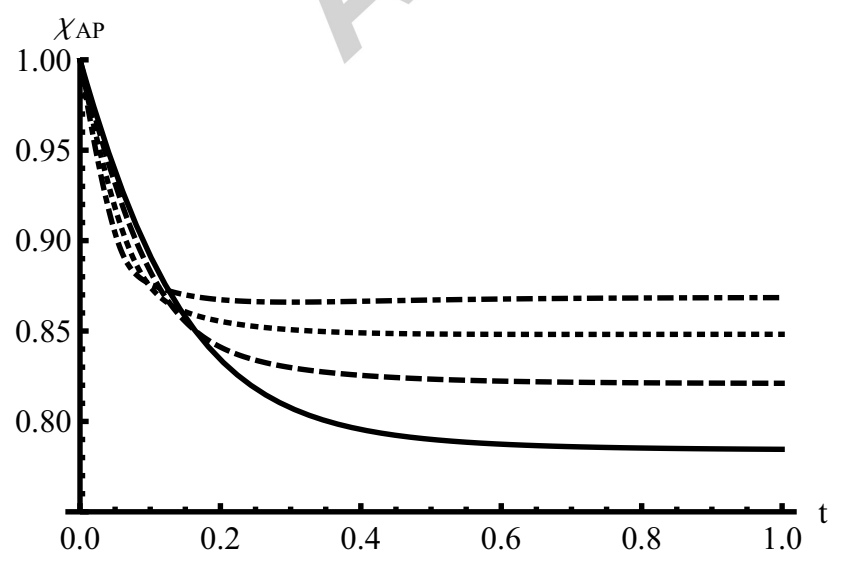

The plots were generated in the same way as for figure 6.3. At the equilibrium, as value of $k$ increases, $A P$ pairs become less correlated (the curves approach one from below). However, $A P$ pairs always stay correlated. In principle, for a closed system, if the number of $A$ molecules is large, the number of $P$ molecules should be small, and vice versa.

\subsection{Figure 6 - Eigenvalues dependence on $a_{0}$}

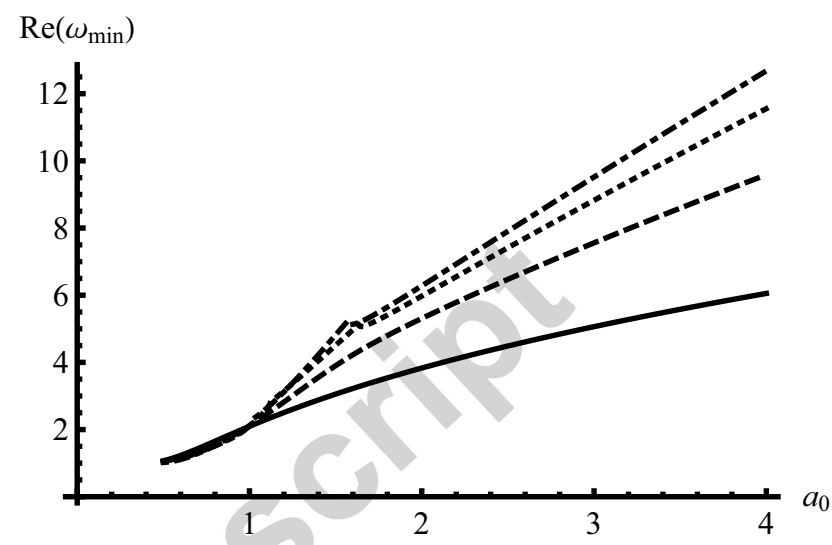

The plots were generated by keeping the initial numbers of $A$ and $P$ particles identical $\left(a_{0}=p_{0}\right)$. All curves were generated by the same ratio of backward and the forward rate, taken to be $\delta / \lambda=1$. The series of $k$ values used is $k=2$ (full line), 3 (dashed line), 4 (dotted), 5 (dot-dash). The approach to the stationary state is speeded up by increasing $a_{0}$. For large $k$ values the equilibration process is faster.

\subsection{Figure 7 - Eigenvalues dependence on $k$}

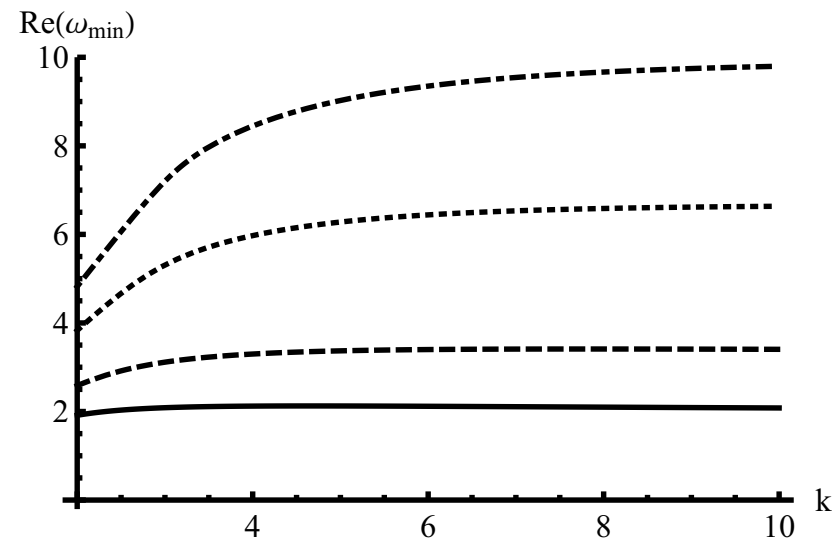

The plots were generated for $a_{0}=p_{0}=2$ and the series of $\delta / \lambda$ ratios; 0.3 (full line), 0.5 (dashed line), 1 (dotted), 1.5 (dotdashed). The approach to the stationary state happens faster for large $k$ values. The effect is less pronounced for low $\delta / \lambda$ ratios. Also, the increase in the $\delta / \lambda$ ratio speeds up the equilibration process. 
6.8. Figure 8 - AA pairs correlation function for open system

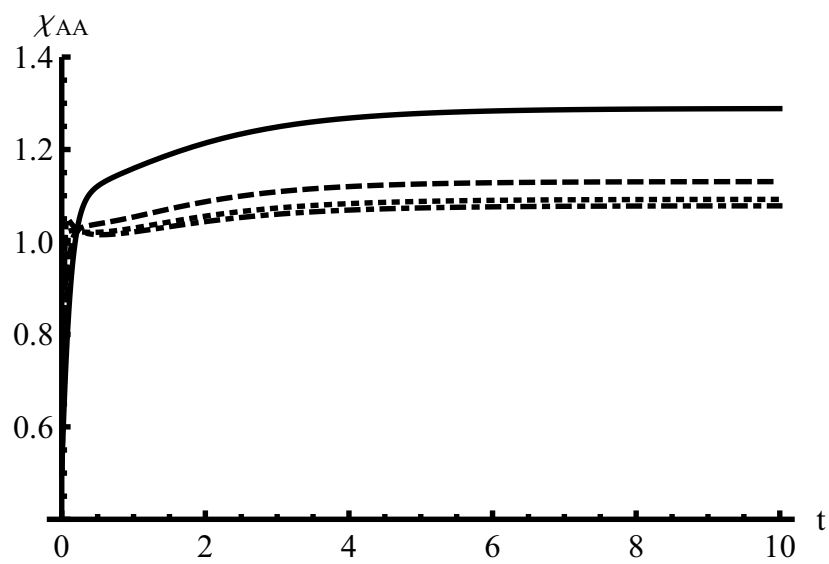

The plots were generated for $\delta / \lambda=1, j / \lambda=1, \gamma / \lambda=1$, $a_{0}=p_{0}=2$ and $k=2$ (full line), 3 (dashed line), 4 (dotted line), and 5 (dot-dashed line). For large $k$ values all curves approach one and the stationary state distribution becomes more Poisson like. A similar behavior was observed for the closed system (please compare with Fig. 6.3). Opening the system increases the overall noise level in the number of $A$ particles. All curves are above one, which can be contrasted with the curves for the closed system which stayed below one.

\subsection{Figure 9 - PP pairs correlation function for open system}

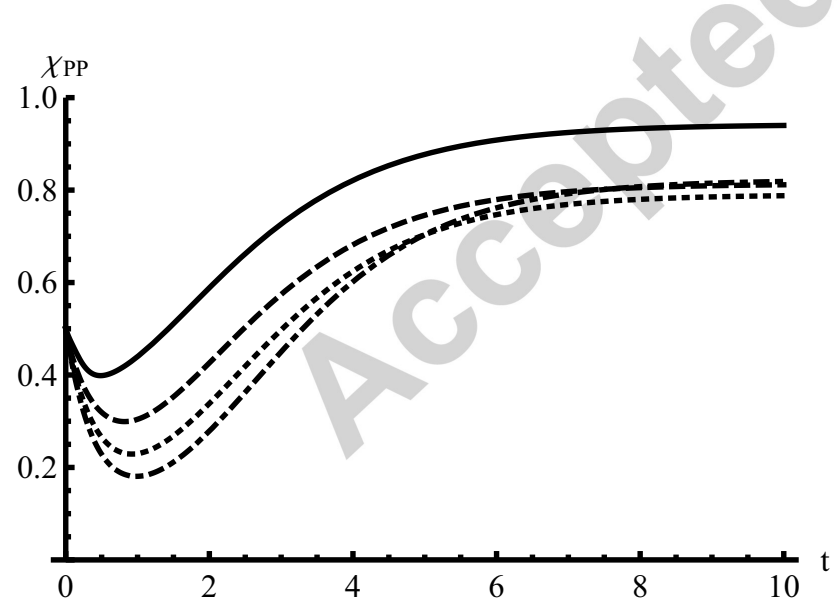

The plots were generated in the same way as for figure 6.8. In contrast to the $A A$ pair correlation function, the $P P$ pair correlation function describes larger deviation from the Poisson distribution for large $k$ values. The same behavior was observed for the closed system (please compare with Fig.6.4). Amount of the noise is reduced for large $k$ values. However, the overall noise level increases by opening the system (all curves move upwards closer to one).

\subsection{Figure 10 - AP pairs correlation function for open system}

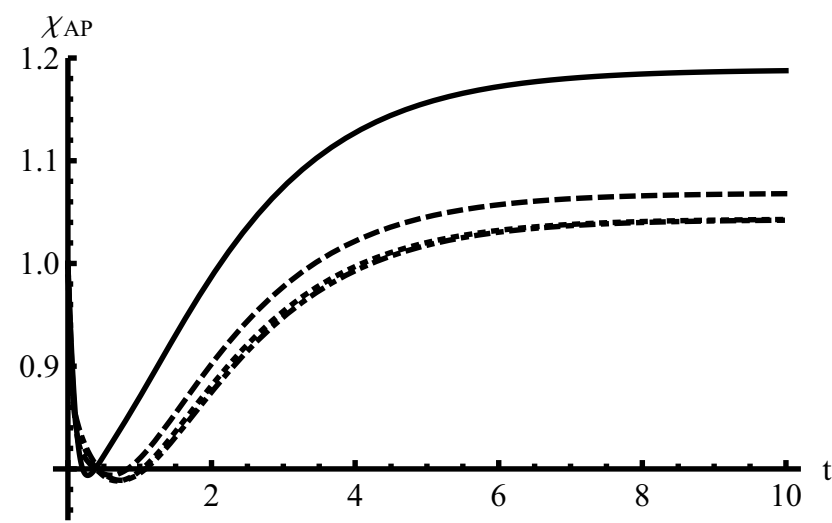

The plots were generated in the same way as for figure 6.8 . At the stationary state, as value of $k$ increases, $A P$ pairs become less correlated. Similar behavior was observed for the closed system (please compare with Fig. 6.5), where opening the system reduced the amount of the correlation since particles are injected at random. However, $A P$ pairs stay weakly correlated, though in a different way (all curves are above one while for the closed system all curves are below one).

\section{References}

[1] C. V. Rao, D. M. Wolf, A. P. Arkin, Control, exploitation and tolerance of intracellular noise, Nature 420 (6912) (2002) 231-237.

[2] R. Grima, S. Schnell, Modelling reaction kinetics inside cells, in: Essays in Biochemistry: Systems Biology, Vol 45, Vol. 45 of Essays in Biochemistry, Portland Press Ltd, London, 2008, pp. 41-56.

[3] J. Elf, M. Ehrenberg, Fast evaluation of fluctuations in biochemical networks with the linear noise approximation, Genome Research 13 (11) (2003) 2475-2484.

[4] C. A. Gomez-Uribe, G. C. Verghese, Mass fluctuation kinetics: Capturing stochastic effects in systems of chemical reactions through coupled mean-variance computations, Journal of Chemical Physics 126 (2) (2007) 024109 .

[5] R. Grima, Investigating the robustness of the classical enzyme kinetic equations in small intracellular compartments, Bmc Systems Biology 3 (2009) 101

[6] E. M. Ozbudak, M. Thattai, I. Kurtser, A. D. Grossman, A. van Oudenaarden, Regulation of noise in the expression of a single gene, Nature Genetics 31 (1) (2002) 69-73.

[7] A. C. Nica, E. T. Dermitzakis, Using gene expression to investigate the genetic basis of complex disorders, Human Molecular Genetics 17 (2008) R129-R134.

[8] P. Stange, D. Zanette, A. Mikhailov, B. Hess, Self-organizing molecular networks (vol 72, pg 73, 1998), Biophysical Chemistry 75 (2) (1998) $161-162$.

[9] M. Wenner, Bring in the noise, Scientific American 299 (1) (2008) 20-+.

[10] H. Kuthan, Self-organisation and orderly processes by individual protein complexes in the bacterial cell, Progress in Biophysics and Molecular Biology 75 (1-2) (2001) 1-17.

[11] B. P. Lee, Renormalization-group calculation for the reaction $\mathrm{ka} \rightarrow 0$, Journal of Physics a-Mathematical and General 27 (8) (1994) 2633-2652.

[12] J. N. Weiss, The hill equation revisited: uses and misuses, Faseb Journal 11 (11) (1997) 835-841

[13] L. Leyton, A. F. G. Quest, Supramolecular complex formation in cell signaling and disease: an update on a recurrent theme in cell life and death, Biological Research 37 (1) (2004) 29-43.

[14] J. R. Williamson, Cooperativity in macromolecular assembly, Nature Chemical Biology 4 (8) (2008) 458-465.

[15] A. Radhakrishnan, X.-M. Li, R. E. Brown, H. M. McConnell, Stoichiometry of cholesterol-sphingomyelin condensed complexes in monolayers, 
Biochimica et Biophysica Acta (BBA) - Biomembranes 1511 (1) (2001) $1-6$.

[16] L. Matthews, G. Gopinath, M. Gillespie, M. Caudy, D. Croft, B. de Bono, P. Garapati, J. Hemish, H. Hermjakob, B. Jassal, A. Kanapin, S. Lewis, S. Mahajan, B. May, E. Schmidt, I. Vastrik, G. M. Wu, E. Birney, L. Stein, P. D'Eustachio, Reactome knowledgebase of human biological pathways and processes, Nucleic Acids Research 37 (2009) D619-D622.

[17] U. Alon, An introduction to systems biology: Design principles of biological circuits, Taylor and Francis Group, Boca Raton, 2007.

[18] L. Mandel, Sub-poissonian photon statistics in resonance fluorescence, Optics Letters 4 (7) (1979) 205-207.

[19] D. C. Mattis, M. L. Glasser, The uses of quantum field theory in diffusionlimited reactions, Reviews of Modern Physics 70 (3) (1998) 979-1001.

[20] E. Kotomin, V. Kuzovkov, Modern aspects of diffusion-controlled reactions : cooperative phenomena in bimolecular processes, Vol. 34 of Comprehensive chemical kinetics, Elsevier, Amsterdam, 1996.

[21] Z. Konkoli, Application of bogolyubov's theory of weakly nonideal bose gases to the $a+a, a+b, b+b$ reaction-diffusion system, Physical Review E 69 (1) (2004) 011106. 